\title{
Vascular age for predicting cardiovascular risk: A novel clinical marker or just a mathematical permutation
}

\author{
Habib A. Dakik, MD, FACC, FASNC \\ a Department of Internal Medicine, American University of Beirut Medical Center, Beirut, \\ Lebanon
}

Received Jan 27, 2018; accepted Jan 29, 2018

doi: $10.1007 / \mathrm{s} 12350-018-1223-\mathrm{x}$

\section{See related article, pp. 1348-1355}

Assessment of cardiovascular risk is a cornerstone in the evaluation and management of patients with suspected coronary artery disease (CAD). Several risk scores have been developed to estimate this risk which primarily relies on the measurement of traditional risk factors in various populations. ${ }^{1}$ Over the past two to three decades new imaging modalities have emerged that can also predict cardiovascular risk, such as Carotid Intima-Media Thickness (CIMT) and Coronary Artery Calcium (CAC) score. Vascular Age is a new variable that has been proposed for utilization in cardiovascular risk assessment. Several models have been proposed for its calculation. It is primarily a conversion of chronological age to an age modulated by traditional risk factors or by imaging findings such as CAC score or CIMT. $^{2-5}$ However, up till now, there has not been a consensus neither on a unified determination of "vascular age" nor on its role in risk assessment.

In this issue of the journal, Nappi et al. investigated the relationship between Coronary vascular age and myocardial ischemia. ${ }^{6}$ They took 717 patients who were referred for both $\mathrm{Rb} \mathrm{PET/CT}$ myocardial perfusion imaging and CAC scoring. Vascular age was determined from the combination of estimated CAD risk for chronological age and CAC score. Vascular age was

Reprint requests: Habib A. Dakik, MD, FACC, FASNC, Department of Internal Medicine, American University of Beirut Medical Center, Beirut, Lebanon; hd01@aub.edu.lb

J Nucl Cardiol 2019;26:1356-7.

1071-3581/ $\$ 34.00$

Copyright (c) 2018 American Society of Nuclear Cardiology. significantly higher than chronological age in patients with ischemia. On the other hand, it was significantly lower than chronological age in those without ischemia. Using incremental analysis, vascular age was more predictive of the presence of myocardial ischemia than chronological age. What is the clinical relevance of these findings?

Chronological age is an easy and long-standing risk marker that is used intuitively on routine basis whenever we evaluate patients for suspected CAD. Is it a perfect marker? Of course not, just like all other known CAD risk factors. Every physician who has been long enough in clinical practice will remember cases of patients who were old, smokers, diabetic, hypertensive, smokers and yet had no obstructive CAD at all when they had a coronary angiogram. On the other hand, they would also remember young patients presenting with acute myocardial infarction with no risk factors at all and with a CAC score of zero. This implies that our assessment of patients should not simply rely on a marker or a risk model but on sound clinical judgment that is guided (but not completely driven) by all of these risk factors, models, and imaging tools. To come back to the findings of Nappi et al., we know for a long time that chronological age and CAC score are both individual markers of myocardial ischemia. ${ }^{7}$ It is therefore not surprising that a combination of these two markers will be even a better marker of myocardial ischemia. It is also not surprising that the combination of these two markers would be a better marker of cardiovascular risk in general. But does that mean that we should drop chronological age in our routine clinical assessment and replace it with "Vascular age"? This would be a big leap, especially that this argument could be extrapolated to other risk factors as well. A 40-year-old man with diabetes would have a higher cardiovascular risk than a 40-year-old man without diabetes. Does that mean that 
we should have a "Diabetic age" to adjust the age of a patient for the presence or absence of diabetes?

The findings of Nappi et al. are important and add to our understanding of the wide spectrum of coronary atherosclerosis with its risk factors and risk assessment models. However, with the increasing number of these models (363 as indicated by Damen et al. ${ }^{1}$ ) and of the imaging techniques available for the assessment of patients with suspected CAD, it becomes important for the scientific community to decrease the complexity of this assessment and come up with simple algorithms that can guide the practicing physician as well as the patient in the prevention and treatment of cardiovascular disease.

\section{Disclosure}

The author has nothing to disclose.

\section{References}

1. Damen JA, Hooft L, Schuit E, et al. Prediction models for cardiovascular disease risk in the general population: Systematic review. BMJ 2016;353:i246.
2. Stein JH, Fraizer MC, Aeschlimann SE, et al. Vascular age: Integrating carotid intima-media thickness measurements with global coronary risk assessment. Clin Cardiol 2004;27:388-92.

3. McClelland RL, Nasir K, Budoff M, et al. Arterial age as a function of coronary artery calcium (from the Multi-Ethnic Study of Atherosclerosis (MESA)). Am J Cardiol 2009;103:59-63.

4. Cuocolo A, Klain M, Petretta M. Coronary vascular age comes of age. J Nucl Cardiol 2017;24:1835-6.

5. Groenewegen KA, Den Ruijter HM, Pasterkamp G, et al. Vascular age to determine cardiovascular disease risk: A systematic review of its concepts, definitions, and clinical applications. Eur J Prev Cardiol 2016;23:264-74.

6. Nappi C, Gaudieri V, Acampa W, et al. Coronary vascular age: an alternate means for predicting stress-induced myocardial ischemia in patients with suspected coronary artery disease. J Nucl Cardiol 2018. https://doi.org/10.1007/s12350-018-1191-1.

7. He ZX, Hedrick TD, Pratt CM, et al. Severity of coronary artery calcification by electron beam computed tomography predicts silent myocardial ischemia. Circulation 2000;101:251-5. 